\title{
Player Fitness and Team Squad Selection using Intelligence System in Sri Lanka
}

\author{
Jayaprada A.B.C., \\ Ihalapathirana K.P. \\ Faculty of Computing \\ SLIIT \\ Malabe, Sri Lanka
}

\author{
Anjalie Gamage, Thilini \\ Jayalath \\ Faculty of Computing \\ SLIIT \\ Malabe, Sri Lanka
}

\author{
Jayasundara U.L., Trishan \\ Piyumal M.A. \\ Faculty of Computing \\ SLIIT \\ Malabe, Sri Lanka
}

\begin{abstract}
Cricket is one of the most popular sports in the world, second only to football. Specially in Asian counties there are millions of cricket fans. Cricket can be considered as the most famous sport in Sri Lanka as well. Although there are many other games, cricket takes an important place because of its attractive playing structure. Cricket is globally played 104 members 12 of which are full members who participate in the Test Matches and the other 94 being associate members of the international cricket Council (ICC). As cricket takes a vital place among all other sports, there are lot actions that have been taken by cricket boards in various countries. Unfortunately, current Sri Lankan cricket is not in a very satisfactory level. Cricket board and government take many actions to uplift the Sri Lankan cricket. when talking about systematic ways, unfortunately there are only few systems which have been built to uplift cricket industry. in this research, our main concern is to build a system which helps to uplift the cricket industry, especially in Sri Lanka. Identifying the cricket pitch condition, batting techniques, players fitness levels, match prediction and team squared selection are the main outcomes of this research. Those are the most important aspects which directly effect to the victory of a cricket match. By introducing this system to the market, we hope to uplift the cricket industry in a much efficient way.
\end{abstract}

\section{Keywords}

Image Process, Video Process, Neural Network, Machine Learning, Hybrid Architecture

\section{INTRODUCTION}

Sri Lanka got full membership in 1981. In that sense, Sri Lanka has a proud history of cricket. Sri Lankan cricket team won the 1996 world cup and the 2014 T20 world cup. But all cricket fans are disappointed with the current state of cricket in Sri Lanka. This is because the game of cricket has been in decline for many years. Today, cricket in Sri Lanka is in the deepest abyss. The main reasons for this are poor player management and poor team selection. In this research, we primarily analyse the player's batting techniques and fitness based on video processing and statics. Through this we can directly identify the player's batting shot and accuracy of that shots.it helps to improve his talent by identifying weaknesses at early stage. And, through this research we create a system to find the fitness of the players. That is very important to identify the most suitable players to cricket tournaments. Fitness of the players directly effect to the victory of a match. This will enable us to apply the necessary solutions for the management of the players.
In this research paper mainly focus on 4 Problems of Nowadays Sri Lankan Cricket Team faced. They are, Cricket Pitch Analysis done by using look of the pitch. It is very Traditional Method. Its Accuracy is very Less. And Player Playing Techniques Analysis only using Coaches Experience. Since some of shots accuracy is very poor. Mainly concern problem is Team squad selection. Currently it is depending on cricket committee favouriting and their knowledge. Since those decisions are wrong. Nowadays we can see the current Situation of our Sri Lank Cricket Team. And, currently there have no system to predict who will be the winning team at the begging of the match. That also a problem of the Cricket. When we can identify the beginning and middle of the match who will be the winner of the match, we can get the right decisions for the win the match.

In here we use Deep Learning Techniques to identify about the condition of the pitch. The cricket pitch is an especially important a part of this sport. The pitch plays a vital role in cricket. There are several pitches that helps bowling and bating. The condition of the pitch plays a significant role within the determination of the game. Once winning the toss the team captain chooses bating first or bowling first. Mostly it's depending on the pitch. Prior to selecting, the team captains of each group can check the pitch completely. Generally, it's terribly tough a play on some cricket pitches. So, according to our Research hope to Classify pitch by using Pitch Images and Historical Weather Data. When Pitch Analysis using image processing mainly used Convolutional Neural Network. And, When the Weather Classify for prediction mainly used Decision Tree Algorithm for that prediction. When both 2 Models combined with together and finally get out put using Ensemble Technique as weighted Average. Additionally, when the user uses the user can change the weight as their want. [3]

And in this research, we use video processing to identify the batting shots of the players. Video processing technology helps to recognize and spot the correct body angles and movements and through that it is easy to understand the most skillful players to the team. And that directly combined with the victory of the match. Getting the percentage of the shot accuracy, can be taken by analyzing the hand movements, leg movements, angel of the players elbow, angel of his torso and the way he directs the bat. There are many other measurements that can be identified using video processing and using those technologies system will identify the shot that a player is playing.

And, here we create a system to identify players fitness as well as team selection. Analyze player's fitness level, Analyze Player's captaincy skill and categorize them to percentage of wining as captain, Categorize Batman's, bowlers, allrounders and wicket keepers according to their performance, calculate 
opposition team's strength according to their players' performance and historical data, analyze important level and difficult level according to opposition team and playing match also include in this system. Another main part we built here Match prediction using machine learning based on historical data. Match prediction system will help to get a clear idea about which team has the highest possibility to win the match. This will be helpful for the coaches too, as they can take necessary actions and give necessary advice for the team by understanding, which team is going to lose, and which team is going to win. The main concern of this research is to uplift the cricket industry in Sri Lanka as the current condition of the Sri Lankan cricket is not in a satisfactory level After This system is introduced to the market, especially young cricket club players can gain so many benefits through this system.

\section{METHODOLOGY}

The focus of achievement of this system is the well cricket player management, Identify the cricket pitch type and select whether the batting or bowling first, Analyse the player playing techniques and match outcome prediction. overview diagram for the proposed system is depicted through figure 1 . This system was designed with the data collection obtained through a survey conducted with a sample group of randomly selected personals from the public.

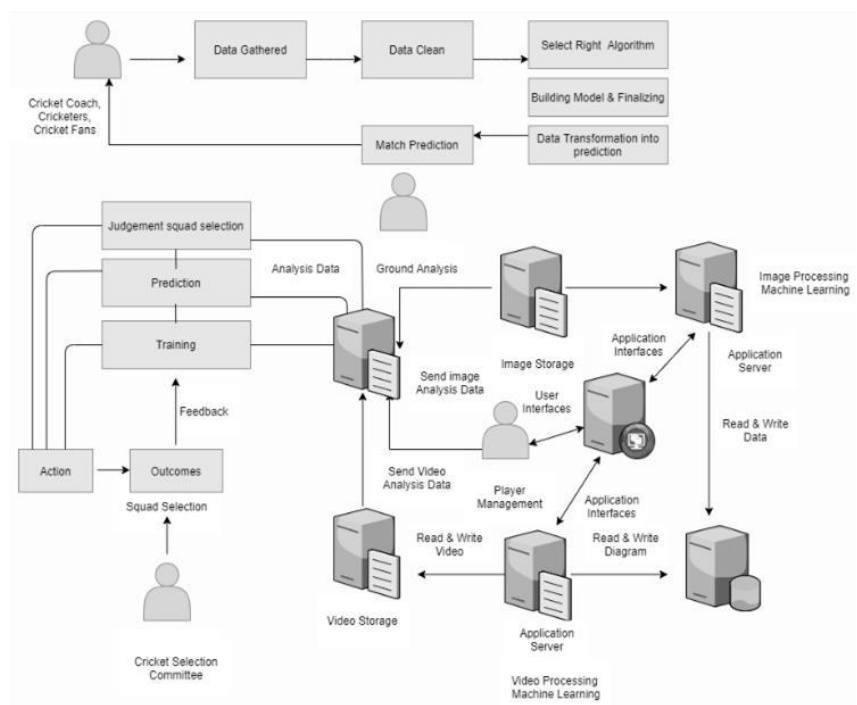

\section{Cricket Pitch Analysis using Deep \\ Learning}

The proposed cricket Pitch Analysis has capability of identifying the Cricket pitch by looking surface. It is most Traditional way to identify the pitch type whether the pitch is Batting pitch or Bowling Pitch. Since, I proposed system for identify pitch Correctly using Deep Learning Techniques. I mention below Figure 1 as proposed system Architecture is Hybrid Architecture model which has two Models and combined those with Ensemble Weighted Average. Technique. Additionally, when

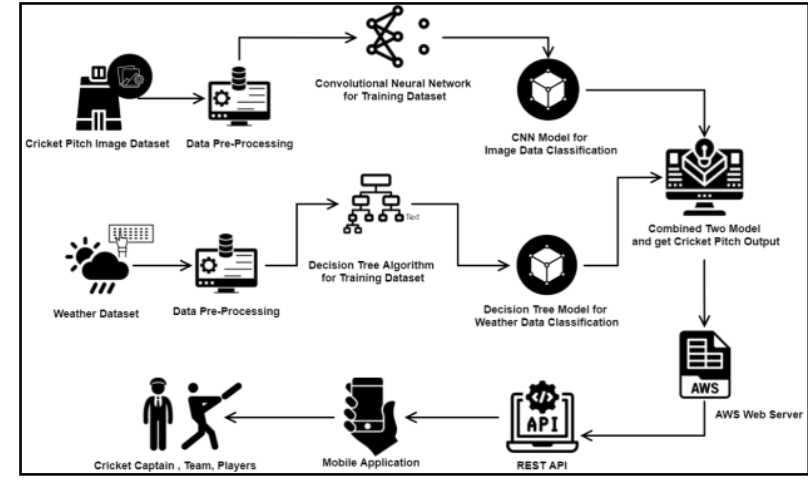

the user uses the user can change the weight as their want.

Figure 1:Hybrid Model Architecture - Ensemble Weighted Averaging

- Data Sources

The data used by the research works examined originated from several sources, i.e., To gather the available weather and environmental data and match toss winning team datasets gather from espncricinfo [7], myweather2 [6], images datasets gather from images capture by field visit \&Kagger [8], Shutterstock. And already collect more than 14000 Images for Training and Validation Dataset. And Collect more than 1000 of Weather details.

\section{- Data Pre-processing}

At this stage removed the noise represented by incorrect and incomplete data to which feature selection was applied. The most common pre-processing procedure was data normalization, which transforms the characteristics into the same scale to enhance the performance.

In this research, I predict whether Pitch is bowling pitch or batting pitch from the Pitch surface images collection sets and weather dataset. Considering the different factors that interact with the Pitch Analysis, and the prediction models can be classified into two main groups based on the input parameters processed:

1.Predict models based on weather data using Decision Tree Algorithm

Before I select Decision Tree Algorithm, compare several machine learning models to get an idea which will be the best. There are,

- $\quad$ Support Vector Regression Model

- K nearest Algorithm Model

- Decision Tree Algorithm Model

- Naïve bayes Classifier Model.

When Considered About these 4 Models the Accuracy are Different. According to the Support Vector Model the Accuracy is 0. 7125.And, $\mathrm{K}$ nearest Algorithm model shows Accuracy as 0. 825.Naive bayes Classifier display 0.55 Accuracy. It is more less than other three. The Best Highest Accuracy Model is Decision Tree Model. Its Accuracy is 0.95. Since I choose Decision Tree Algorithm for Weather Data Classification. Historical weather data as temperature, humidity, rainfall, wind, Cloud, Pressure collected from several locations over 4 years (2012-2019) were analyzed. I hope collect dataset as shown in table 3.1. Weather data is more important for more accurate pitch analysis. In classify weather condition (Batting or Bowling pitch) using Decision Tree is trained using different combinations of weather parameters. When collect weather data, Mainly Focus Weather 
Conditions are, Humidity, Rain, Pressure, Cloud, Wind, Temperature.

2.Predict models based on image processing using Convolutional Neural Network

When I Predict model base on image mainly used Convolutional Neural Network as a Technology. According to the CNN I follow some steps to done of image prediction. We need three basic components to define a basic convolutional network as shown

Figure 2. They are,

- The convolutional layer - In this layer, the mathematical operation of convolution is performed between the input image and a filter of a particular size

MxM.

- The Pooling layer[optional] - In this layer decrease the size of the convolved feature map to reduce the computational costs.

- Fully Connected Layer - The Fully Connected (FC) layer consists of the weights and biases along with the neurons and is used to connect the neurons between two different layers.

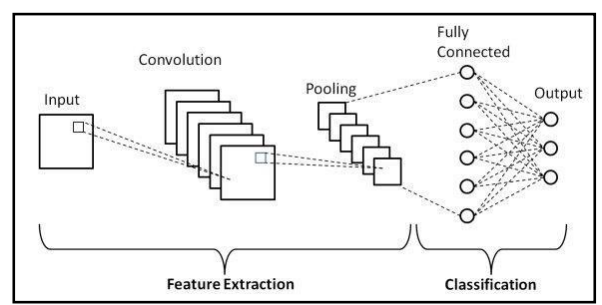

Figure 2:Convolutional Neural Network Architecture

3.Combine 2 method using Ensemble method as Weighted Average Ensemble

When combined two methods for the final output of pitch type. Since I used Ensemble Method as Weighted Average Ensemble. This method weights the contribution of each ensemble member based on their performance on a hold-out validation dataset. Models with better contribution receive a higher weight.

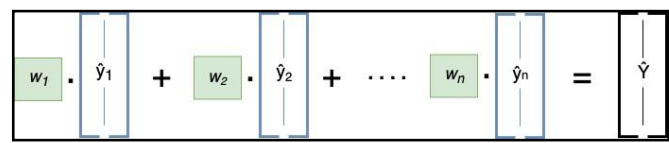

Figure 3:Weighted Average Ensemble is all about weighting the predictions of each base-model generating a combined prediction

Here we have Model1 to Model 2 individual models which are giving predictions from Pred 1 to Pred 2 respectively. And we combine them to come up with a single model. we have two models, M1, M2, which are based on different techniques such as first could be based on CNN and second could be based Decision Tree Classifier. And we combine them to come up with a single model. I combination techniques as Weighted Averaging. According to this technique I'm not taking into consideration the accuracy of individual models. I'm giving each of these model's equal weightage, which is not a very prudent strategy because if I know that a model is performing better, I will want to give it higher weightage As I shown above Figure 3.

So, what I've done is I've taken weighted average from these models, but the weights were decided by user. That should give user better results because I understand that these models work better, and I've given them more weight. And I added newly part as weigh can be change manually from our proposed application. So, it will help to user to add the weight according to the situation.

\section{Player Analysis using Video Processing}

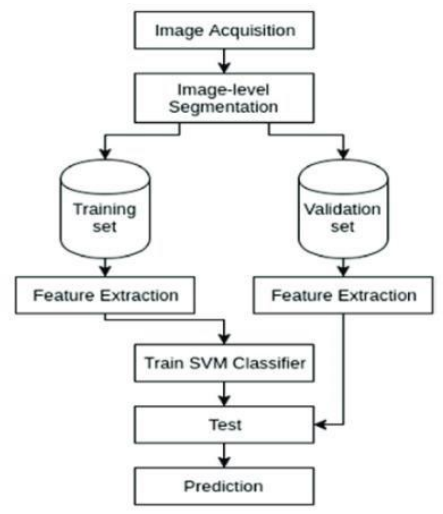

Figure 4:Player Analysis Process

The proposed cricket players batting technique analyser has capability of identifying the player body movements and identify the batting shot which he is performing. This can be described as a motion analysis as well. For this the main technology comes as video processing. System will analyse the videos and produce output with high accuracy, even though the video quality is bad. For that, the algorithms will be implemented in a new way. When analysing the videos there might be various input videos. There can be low quality videos, videos with lighting changes and blurred videos. Here the algorithm will be modified to uplift the accuracy, so that all the results will come with a higher accuracy. With this implementation no matter how low the input video quality is, the output accuracy will be high.

\section{- Data Gathering \& processing}

Gathering data is the most crucial requirement in this research part. Here data has been collected by using real cricket club players. Here I have collected data sets from many individual players and then processed the captured video and prepared the data set. Having the correct data set with the correct amount, directly affects the accuracy of the final outcome. Here $\mathrm{i}$ have used adobe premiere pro to edit and preprocess the videos.

\section{- Used Libraries}

Prior to processing the video clips, the video should break into individual image frames. In order to do that there, I have used NumPy and OpenCV libraries. OpenCV comes with many powerful video editing functions. OpenCV is a free and open-source library that allows us to do a lot of different types of images and video processing. OpenCV, on the other hand, is a good bargain due to its versatility. And also, NumPy has been used here as one of the libraries. NumPy is a Python library that adds functionality for larger, multi-dimensional arrays towards the language [1]. And in this process the image 
will be separated into individual frames and will be stored in the system.

\section{- $\quad$ Methods \& Approach}

Here Keras NumPy, pandas, matplotlib, pylab and seaborn are the main libraries. Pandas is a widely used opensource Python library for data science, data analysis, and machine learning activities. It is built on top of NumPy, a library that supports multi-dimensional arrays. Matplotlib creates highquality figures in a range of hardcopy and interactive formats across different platforms. Matplotlib is a Python library that used in scripts, Python shells,

Web application servers, and graphical user interface toolkits. Another library that is used here is pyLab.The Matplotlib object-oriented plotting package has a procedural interface called PyLab. Here I have used seaborn as well. Seaborn is a Python library that is mostly used to create statistical visuals. Seaborn is a Python data visualization package based on matplotlib that is tightly connected with pandas' data structures.[2] The central component of Seaborn is visualization, which helps in data exploration and comprehension.

\section{- Make the Model}

To make the model, ReLU has been used here. ReLU is a layer activation function in Keras.In deep learning models, the Rectified Linear Unit is the most often used activation function. If the function receives any negative input, it returns 0 ; however, if the function receives any positive value $\mathrm{x}$, it returns that value.

As a result, $y=\max$ can be written $(0, x)$ Some aspects of the ReLU function It's really simple to understand, and it's not based on any hard arithmetic formula [3.] It is free of the dying slope problem that affects other activation functions like sigmoid and Tanh.

And also here i have used SoftMax as well.

\section{- Train the Model}

We can apply pre-trained models from the Keras core library for image recognition. VCG16, VCG19, Resnet50, Inception V3, and Xception models are examples of such models. To train the model I have used xception function mainly in here.

\section{Team Squad Selection \& Player Fitness Analysis using Machine Learning}

\section{- Data Set}

Gather data from Cricinfo website using Parsehub tool. There we collect data as batting information and bowler information. We gathered 38000 bowling information data set and 38000 batting information data sets. Balls played, Player's country, Opposition Country, Strike rate, Average, Ground name, innings runs score, weather,50s and 100s as data types of batting information. Player's country, Opposition Country, Economy rate, Overs bowled, run conceded, taken wickets, Maiden bowled, weather, 3 wickets and 5 wickets as data type of bowling information. Then we import our data set as an excel file and load that data for training our model. Then we did data pre-processing for data cleaning.

Player's performance is more affected for squad selection. Because player's performance is decided match's win or lost. Therefore, player's performance analyze is more valuable for squad selection process. There first we need to categorize current players such as batman, bowler, allrounder and wicket keeper. We can categorize players by using their playing role according to individual player's statics in Cricinfo site. There we consider 5 functions for select 11 players of squad. Figure 5 is shown System architecture diagram for squad selection.

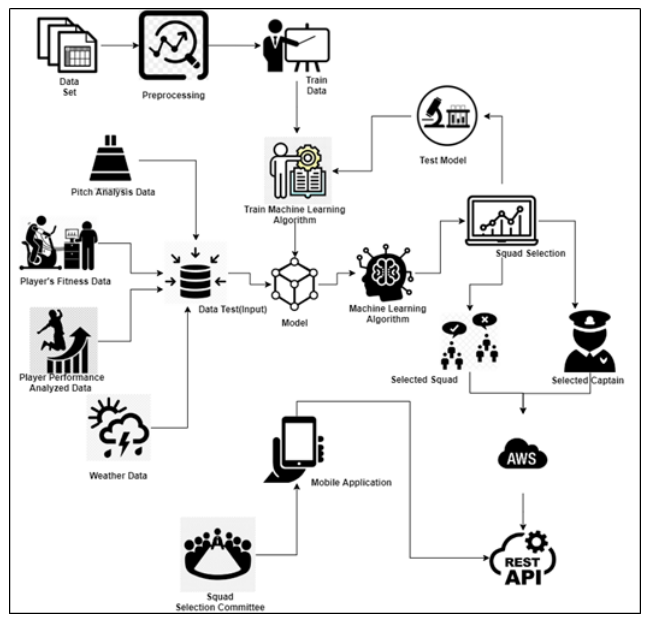

Figure 5:System Architecture Diagram for Squad Selection

\section{Predict models based on weather data using Decision Tree Algorithm}

We analysed batting performances by using player's country, opposition Country, Strike rate, Average, Ground name, innings runs score, weather,50s and $100 \mathrm{~s}$ as data types of batting information. And analysed bowling performances by using. Player's country, Opposition Country, Economy rate, Overs bowled, run conceded, taken wickets, Maiden bowled, weather, 3 wickets and 5 wickets as data type of bowling information. There we measured player's consistency, form, ground strength, and opposition strength. For that we used AHP (Analytic Hierarchy Process). Batting/Bowling consistency means player's experience through his cricket entire carrier. There we measured batting consistency by using player's entire carrier statics. Batting/Bowling Form means player's performance of last year. There we measured batting Form by using player's last year statics. Opposition strength means player's performance against the particular team. There we measured Opposition strength by using player's entire cricket statics against Particular team. Ground strength means player's performance at a particular venue. There we measured Ground strength by using player's entire cricket statics at particular ground.

Batting Consistency $=0.4262 *$ Average +
$0.2566 *$ PlayedInnings
$+\quad 0.1510 *$ StrikeRate $+0.0787 * 100 \mathrm{~s}+0.0556 * 50 \mathrm{~s}-$
$0.0328 * 0 \mathrm{~s}$

Bowling Consistency $=0.4174 *$ Bowled overs + $0.2634 *$ Played Innings $+0.1602 *$ StrikeRate + $0.0975^{*}$ Average $+0.0615^{*}$ Five or Four Wickets

Batting Form $=0.4262 *$ Average $+0.2566 *$ PlayedInnings + $0.1510 *$ StrikeRate $+0.0787 * 100 \mathrm{~s}+0.0556 * 50 \mathrm{~s}-0.0328 * 0 \mathrm{~s}$

Bowling Form $=0.3269 *$ Bowled overs + $0.2846 *$ PlayedInnings $+0.1877 *$ StrikeRate $+0.1210 *$ Average $+0.0798^{*}$ Five or Four

Wickets 
Batting Opposing Strength $=0.4262 *$ Average + $0.2566 *$ Played Innings $+0.1510 *$ StrikeRate $+0.0787 * 100 \mathrm{~s}+$ $0.0556 * 50 \mathrm{~s}-0.0328 * 0 \mathrm{~s}$

Bowling Opposing Strength $=0.3177 *$ Bowled overs + $0.3177 *$ PlayedInnings $+0.1933 *$ StrikeRate $+0.1465 *$ Average $+$

0.0943* Five or Four Wickets.

Batting Ground Strength $=0.4262 *$ Average $+0.2566 *$ Played Innings $+0.1510 *$ StrikeRate $+0.0787 * 100 \mathrm{~s}+0.0556 * 50 \mathrm{~s}+$ $0.0328 * \mathrm{HS}$

Bowling Ground Strength $=0.3018 *$ Bowled overs + $0.2783 *$ PlayedInnings $+0.1836 *$ StrikeRate $+0.1391 *$ Average

0.0972* Five or Four Wickets.

Then we predicted player's batting next match score and bowler's next match taking wickets by using random forest classifier, Naïve bayes and decision tree algorithm. Then we chose the most accurate model/algorithms among them. And we deployed chosen model and combined with android application together.

\section{Wicketkeeper's performance Analyze}

There, categorized wicketkeeper's performance is analyzed by us. There we consider about wicketkeeper's dismissals, batting average, batting strike rate and last five matches' dismissals, batting average, batting strike rate analyzed for wicketkeeper's performance. Then we predict player's upcoming wicket keeping performance for using ML (Machine Learning) algorithm.

Finally, we rank-up wicket keepers according to prediction.

\section{Select captain for Team}

There we choose captain of the team from selected 11 players. There we considered players individual performance, winning percentage of participated matches or captained matches. Then we analyzed that information and give value of each player. Then we choose captain as highest valued player.

\section{Analyze Fitness level of Players 5.}

There we gather player's last five matches player's performance in Cricinfo web site. There we measured player's overall average within last five matches. Then we get player's measured time records by run three runs on a normal length cricket pitch with a bat but no other equipment (pads, gloves etc.). Then we predict fitness percentage by using machine learning algorithm according to overall player's performance average and percentage of running measurement fitness.

\section{Select players for Match}

There we will hope to select 11 players for match victory. There, first we determined number of batsmen, number of spinners, number of fast bowlers and all-rounders according to pitch condition. Then we select 11 players according difficult of match, importance of match, ranked-up categorized players and players ratio. There we select best, good, and normal squad above mentioned matter.

\section{Match Outcome Prediction using Machine Learning}

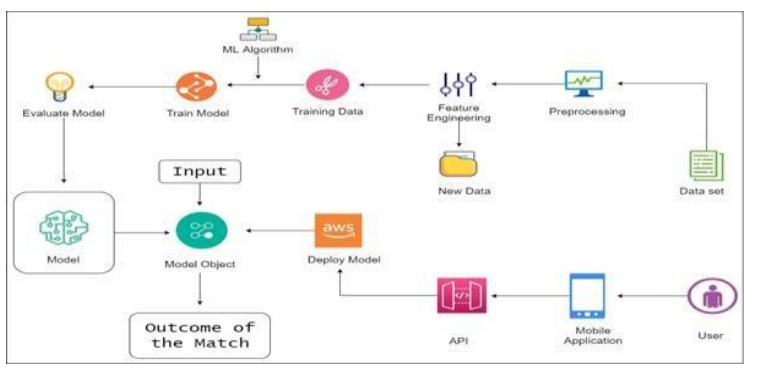

Figure 6:System Overview

- Data source

All the records and datasets that we want are available at one place. We can get those records from ESPN Cricinfo site. The site gives us ball by ball all relevant details of matches including historical data. Also, the most important thing is that a Cricinfo[7] web site is the most trusted web site. Therefore, we can get more accurate data easily from that.

- $\quad$ Proposed Methodology

To predict the outcome of a cricket match I went through the following general Machine Learning workflow step-by step.

1. Data cleaning and formatting

2. Feature engineering and selection of the features that we consider here.

3. Compare several machine learning models to get an idea which will be the best.

4. Evaluate the best model.

5. Using a selected model predicts the outcome of the winning team more accurately.

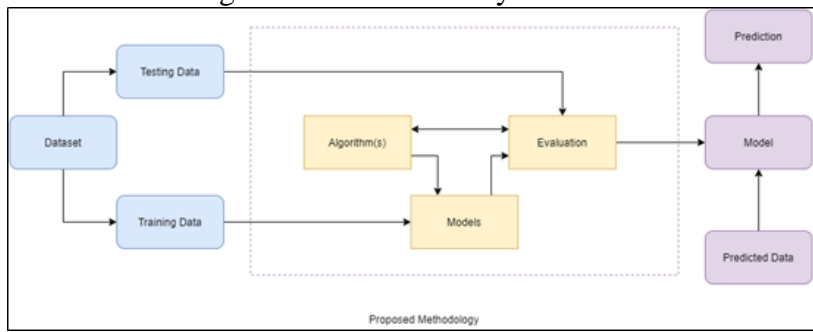

Figure 7:Proposed Methodology

(Figure 7) First I extracted relevant data from the above identified data source. Then I remove unwanted data from the extracted data set. That procedure is identified as data cleaning. Then went through the select important records from the cleaned data set. That procedure is identified as feature selection. Since the algorithm is totally dependent on how we feed data into it, feature engineering should be given utmost priority for every machine learning project. Then select a data set transformed as a suitable data type for prediction. After the data transformation, predict the winning team by using several machine learning models such as Decision Tree Classifier, Random Forest Regressor, Naïve Bayes. Then I measured the accuracy of the algorithm by using my own function. After that, I selected the most accurate model as a Random Forest Regressor which gives the custom accuracy $97.7 \%$.

Model 1 - This model for the ongoing cricket match and here considered following key features,

- Venue - If a team is playing at their home ground, then that team has a better chance to win as they are known to pitch condition, climate and also crowd 
support plays an important role to boost the confidence of players.

- Batting Team, Bowling Team,

- Runs in current situation,

- Wicket in current situation,

- How many runs get the last 5 overs - In here I calculated how many runs got in the last five overs because it will make more sense instead of just using the current score. Such a situation our prediction accuracy will slightly decrease. With these help we can avoid such a situation.

- Also consider How many wickets fallen in the last 5 overs same as previous this will also be important in order to get the best outcome also.

- Striker score, Non-striker score as well

Model II - This model is mainly built for future matches which have already been announced. For example, the upcoming T20 world cup squad was released a few days ago. So, we can predict the outcome before participating in the match. This will enable more advantages to the Sri Lanka selection committee when preparing the squad. Considered,

1. Batting Average of Team 1 - First calculate each player's average as follow,

Player 1 batting average $=$ Total Number of runs/ Total number of times gets out.

Calculate the average of each 11 of players by using the above-mentioned formula. Then, we calculated Batting average of the Team 1 as follow,

Batting Average $=$ Total of all player's batting average/

11

2. Batting Average of Team 2 - Same as the previous step.

3. Bowling Average of Team 1 - First calculate each player's average as follow,

Player 1 bowling average $=$ Total Number of runs/ Total numbers of wickets taken.

Calculate the average of all bowlers by using the abovementioned formula. Then, we calculated Bowling

average of the Team 1 as follow,

Bowling Average $=$ Total of all bowler's bowling average/ Number of bowlers in squad.

4. Bowling Average of Team 2 - Same as the previous step.

Then pickling the above models is stored as a pickle file. As the final step, deploy those models on cloud by using Azure.

\section{RESULT AND DISCUSSION}

Table 1. Testing Summary

\begin{tabular}{|c|c|c|c|}
\hline Scenario & Input & Output & Pass/Fail \\
\hline $\begin{array}{c}\text { Cricket } \\
\text { pitches } \\
\text { predict }\end{array}$ & $\begin{array}{c}\text { Image of the } \\
\text { cricket pitch, } \\
\text { weather details } \\
\text { inputs }\end{array}$ & $\begin{array}{c}\text { Weather and } \\
\text { image prediction } \\
\text { as percentage } \\
\text { (display type of } \\
\text { pitch) }\end{array}$ & Pass \\
\hline
\end{tabular}

\begin{tabular}{|c|c|c|l|}
\hline $\begin{array}{c}\text { Match } \\
\text { prediction } \\
\text { outcomes }\end{array}$ & $\begin{array}{c}\text { Batting and } \\
\text { bowling } \\
\text { average }\end{array}$ & $\begin{array}{c}\text { Match winning } \\
\text { team }\end{array}$ & Pass \\
\hline $\begin{array}{c}\text { Team } \\
\text { squad } \\
\text { selection }\end{array}$ & $\begin{array}{c}\text { Bowling, } \\
\text { batting, ground } \\
\text { details }\end{array}$ & $\begin{array}{c}\text { Team squad } \\
\text { selected list }\end{array}$ & Pass \\
\hline $\begin{array}{c}\text { Player } \\
\text { analysis }\end{array}$ & $\begin{array}{c}\text { Video of the } \\
\text { player playing }\end{array}$ & $\begin{array}{c}\text { Accuracy of the } \\
\text { Type of playing } \\
\text { technique }\end{array}$ & Pass \\
\hline
\end{tabular}

Analyzingindividual player's playing techniques and fitness gives us necessary solutions for the management of the players. By using video processing methods, we analyze those techniques in a more accurate way. Using the final output, there is the ability to identify what are the weak points of the players, and how to train them in better ways. And can get an idea about the shots that a player can perform better. So, the player can gain a high score in a match if he plays that match using those batting shots.

Also, our research gives more accurate cricket pitch analysis, and we can check whether the pitch is a batting pitch or bowling pitch. We demonstrated our new method since the existing method was a manual pitch analysis system with less accuracy. In order todo pitch analysis, we used the Deep Learning Techniques as Convolutional Neural Network for Image Prediction and Decision Tree Classifier for Weather Prediction and Combined those two with Ensemble Weighted Average Technique. Additionally, User Can Update weigh by using Proposed Application. and it will be very helpful to the team which won the toss, to decide whether bat or ball first within predicting data.

Another main result that we gained from this research is by analysing historical data, we provide the best squad for a given opposition team. So here we test several machine learning models and choose the best model with high accuracy which was given over $90 \%$ of accuracy.

Another key feature which included our research was we provide the prediction system as well with high accuracy. Match prediction system will help to get a clear idea about which team has the highest possibility to win the match. In order to approach this, we built a machine learning model which was given over $90 \%$ of accuracy as well as the previous one. It will help team management in preparing the team for the future match.

Most people in the world heard the Sri Lanka name because of Cricket. As cricket takes an indispensable place among all other sports in Sri Lanka, there are a lot of actions that must be taken to improve Sri Lanka cricket. Unfortunately, current Sri Lankan cricket is not at a firing level as we expected. What we expected here is to bring back to Sri Lankan cricket by giving technical support as we can. So, we come up with new functionalities which are very important and sophisticated.

\section{CONCLUSION}

In this research, we primarily analyze the individual player's playing techniques and fitness based on video processing and statics. This will enable us to apply the necessary solutions for the management of the players. Further, ground analysis is largely based on the experience of cricket players and coach. Its accuracy is less than $70 \%$. Therefore, we use image processing technology and historical data analysis for a more accurate Cricket pitch analysis. This allows for a more efficient and accurate higher than manual pitch analyze report. Also, the main reason for the current wave of defeats in the Sri Lankan cricket team is poor selection committee decisions.[1] 
In this case, the decisions of the selection committee are weakened due to the lack of consideration for the capabilities and strength of the opposing team, the lack of a definite assessment of the strength and ability of their team and the fact that the team does not change according to the behavior on the ground. Therefore, our aim is to study the strength and ability of the opposing team and to select the most suitable team to win the competition using machine learning using AI technology and Cricket Pitch analysis, thereby minimizing weak selection committee decisions. And it is hard to even think of winning or losing a game like cricket. Therefore, our goal is to make match predictions using machine learning based on historical data, match simulation, whether team composition. Accordingly, this match prediction system helps to make the necessary decisions now of playing to win the match. There are some main objectives as summarize in our research.

1. Analyzing cricket pitch, individual players playing techniques and fitness. Selecting suitable team squad using the above details and predicting the winning team.

2. Analyze individual player's playing techniques and fitness. This will enable us to apply the necessary solutions for the management of the players.

3. Use image processing technique to analyze for a more accurate Cricket pitch analysis. By analyzing the pitch, we can tell whether the pitch is a batting pitch or bowling pitch. Select best Team squad selection is done by analyzing the historical data and analyzing the strengths and weaknesses of the players.

4. Perform prediction of future matches and helps team management in preparing the team for the future.

\section{FUTURE WORK}

The paper looked ground analyses using image processing. There we did ground analyzing by using images. We propose to ground analyses by using sound tracking and the nature of soil in pitch. Because we couldn't measure nature of the pitch by using only images of pitch.

Ground analyses accuracy is increased when using soundtrack of pitch and the nature of soil in pitch. There we used video processing technique for only analyses batting techniques. We proposed to analyze bowling techniques by using video processing in near future. future. And then we can analyze player's performance very accurately. Also, there we didn't consider about left right batting combination to select squad. For that we need to experience knowledge of cricket match. Therefore, we consider add that feature for squad selection in near future.

\section{REFERENCES}

[1] Cricket Mastery. 2021. How to Read a Cricket Pitch? Avoid Bad Decisions with These Tips! | Cricket Mastery.
[online]Availableat: <https://cricketmastery.com/howtoread-a-cricket-pitch/> [Accessed 18 February 2021]

[2] Ijsr.net. 2014. Cricket Pitches - Science behind the Art of Pitch Making. [online] Available at:

<https://www.ijsr.net/archive/v3i7/MDIwMTU4.pdf> [Accessed 20 February 2021].

[3] ResearchGate. 2020.PredicitngBowling Performance in Cricket from publicity Available Data.

[online]Availableat:<https://www.researchgate.net/publication 1340070875_Predicting_Bowling_Performance_in_Crick et_from_Publicly_Available_Data> [Accessed 22 February 2021].

[4] Meraj-Bin-Malek, Rakib Hasan Badhan, Mohaiminul Islam Shesir, Nazmul Haque Fakir (2018) Squad Selection for Cricket Team Using Machine Learning Algorithms, Department of Computer Science and Engineering BRAC University, Dhaka - 1212, Bangladesh: Mr. Hossain Arif .

[5] Research Gate. 2019. Image Processing Techniques for Detecting and Classification of Plant Disease: A Review. [online]Availableat:<https://www.researchgate.net/public ati

on/334314928_Image_Processing_Techniques_for_Detec ti ng_and_Classification_of_Plant_Disease_A_Review> [Accessed 24 February 2021].

[6] Myweather2.com. 2021. Weather forecast for Galle Stadium| Cricket Ground | Sri Lanka. [online]

Availableat: $<$ https://www.myweather2.com/Cricket/SriLanka/Galle-Stadium.aspx> [Accessed 20 March 2021].

[7] Cricinfo.2021. Match results. [online] Available at: <https://stats.espncricinfo.com/ci/engine/stats/index.html ?cl ass $=1$; host $=8$; result $=1$;result $=2 ;$ result $=3$; result $=4$; templat $\mathrm{e}=$ results;type $=$ allround; view $=$ results $>$ [Accessed 10 February 2021].

[8] Kaggle.com. 2020. Best Batsman+Bowlers-toss-position. [online]Availableat:<https://www.kaggle.com/doosrijana $\mathrm{m} /$ best-batsman-bowlers-toss-position $>$ [Accessed 12 March 2021].

[9] Shutterstock.com. 2021. Cricket Pitch Images, Stock Photos \& Vectors | Shutterstock. [online] Available

at:〈https://www.shutterstock.com/search/cricket+pitch> [Accessed 21 February 2021].

2021. cricket pitch images. [online] Available at: $<$ https://www.dreamstime.com/photosimages/cricketpitch .html $>$ [Accessed 21 February 2021]. 